\title{
Catalogue of solar type II radio bursts observed from September 1990 to December 1993 and their statistical analysis ${ }^{\star}$
}

\author{
G. Mann ${ }^{1}$, A. Klassen ${ }^{1}$, H.-T. Classen ${ }^{1}$, H. Aurass ${ }^{1}$, D. Scholz ${ }^{1}$, R.J. MacDowall ${ }^{2}$ and R.G. Stone ${ }^{2}$ \\ 1 Astrophysikalisches Institut Potsdam, Observatorium für solare Radioastronomie, D-14552 Tremsdorf, Germany \\ 2 NASA/Goddard Space Flight Center, Greenbelt, MD 20771, U.S.A.
}

Received July 20, 1995; accepted March 26, 1996

\begin{abstract}
Solar type II radio bursts represent the radio signature of shock waves travelling through the solar corona. They are associated with flares, coronal mass ejections (CME's) and interplanetary shocks. Type II radio bursts appear as emission stripes slowly drifting from high to low frequencies in dynamic radio spectra. The spectral features of all solar type II radio bursts observed by the new radiospectrograph of the Astrophysikalisches Institut Potsdam in Tremsdorf during the time period from September 1, 1990 to December 31, 1993, i.e., during the first part of the ULYSSES spacecraft mission, are summarized and statistically investigated.
\end{abstract}

Key words: Sun: activity; radio radiation

\section{Introduction}

Wild \& McCready (1950) first reported the observations of so-called type II bursts occurring in nonthermal solar radio radiation. In dynamic radio spectra a type II burst appears as an emission band slowly drifting from high to low frequencies. An example of such a type II radio burst is presented in Fig. 1. These bursts are interpreted as the radio signature of shock waves in the solar corona. Such shock waves can be generated by flares and/or coronal mass ejections (CME's) (Roberts 1959; Smerd et al. 1975; Wild et al. 1972) (cf. also Dryer 1982; Nelson \& Melrose 1985; Bougeret 1985; Aurass 1992; Mann 1995 as reviews). Sometimes coronal shock waves are able to penetrate into interplanetary space where they appear as interplanetary shocks. These interplanetary shocks may emit radio waves observed as interplanetary type II radio bursts observed below $1 \mathrm{MHz}$ (Cane et al. 1982; Stone et al. 1984; LengyelFrey \& Stone 1989).

Generally, solar type II radio bursts consist of two components, i.e., the so-called "backbone" and "herringbones" (cf. e.g. Krüger 1979). The "backbone" is the slowly drifting emission band. The drift rate is roughly $-0.1 \mathrm{MHz} / \mathrm{s}$ (Nelson \& Melrose 1985; Urbarz 1990). Most of the "backbones" show a fundamental-harmonic structure (Nelson \& Melrose 1985). In rare cases the third harmonic band is also visible (cf. Fig. 1) (Roberts 1959; Kliem

Send offprint requests to: G. Mann

${ }^{\star}$ Tables also available in electronic form via anonymous $\mathrm{ftp}$ cdsarc.u-strasbg.fr or ftp 130.79.128.5 et al. 1992; Aurass et al. 1995). Furthermore, the "backbone" has a great variety of fine structures, e.g., band splitting, multiple lane structures etc. (McLean 1967; Wild \& Smerd 1972; Smerd et al. 1975; Nelson \& Robinson 1975; Nelson \& Melrose 1985). The "herringbones" are rapidly drifting emission stripes extending to high and low frequencies from the "backbone" in dynamic radio spectra. Twenty percent of all type II bursts are accompanied by "herringbones" (Roberts 1959). They resemble solar type III radio bursts and are interpreted as the radio signature of electron beams accelerated at the shock wave associated with the type II burst (Nelson \& Melrose 1985). Cairns \& Robinson (1987) deduced that these electrons have velocities in the range $0.08-0.4 c$ ( $c$, velocity of light).

Generally, it is assumed that the radio radiation is generated by suprathermal and/or high energy electrons. These electrons excite high frequency electrostatic plasma waves, i.e., upper hybrid waves or Langmuir waves, which scatter on ion density fluctuations and/or low frequency plasma waves and, thus, are converted into electromagnetic (radio) waves escaping the solar corona. Thus, the emission occurs slightly above the local electron plasma frequency. Furthermore, high frequency electrostatic waves can nonlinearily interact with themselves, coalescing into electromagnetic (radio) waves with frequencies approximately twice the electron plasma frequency. Thus, the former and latter mechanisms are responsible for the fundamental and harmonic radiation, respectively (cf. e.g. Krüger 1979). Benz \& Thejappa (1988) argued 
Fig. 1. Solar type II radio burst observed in the range 40-800 MHz on September 27, 1993. It started at 12:10:00 UT. In this case the fundamental, first and second harmonic band of the "backbone" are clearly visible. The colour scale on the top panel indicates the intensity of the received radio radiation

that the radiation mechanism of the "backbone" is different from that of the "herringbones".

Ginzburg \& Zheleznyakov (1958) were the first to present a type II burst model based on electron streams accelerated at the shock front. Furthermore, Zheleznyakov (1965) and Zaitsev (1966) proposed that the electric current induced within the shock front becomes unstable and, subsequently, excites Langmuir waves needed for type II burst emission. Later on, Holman \& Pesses (1983) and Benz \& Thejappa (1988) suggested that solar type II radio bursts are generated by supercritical, quasi-perpendicular, fast magnetosonic shock waves in the corona. On the other hand, Mann \& Lühr (1994) recently proposed a mechanism for producing solar type II burst radiation at supercritical, quasi-parallel shock waves.
In this paper the spectral properties of all solar type II radio bursts observed during the time period from September 1, 1990 until December 31, 1993 (cf. Table 1), i.e., since the launch of the ULYSSES satellite are reported. The observations were obtained with the new radiospectrograph of the Astrophysikalisches Institut Potsdam in Tremsdorf. It consists of four sub-spectrometers working in the ranges $40-100 \mathrm{MHz}, 100-170 \mathrm{MHz}, 200-400 \mathrm{MHz}$ and $400-$ $800 \mathrm{MHz}$ with a sweep rate of $10 \mathrm{~Hz}$. Further details about this instrument are given by Mann et al. (1992). Comprehensive studies of observations of solar type II bursts and CME's (Gosling et al. 1976; MacQueen 1980; Wagner 1980; Sheeley et al. 1984; Robinson et al. 1984) showed that CME's with a velocity greater than $400 \mathrm{~km} / \mathrm{s}$, which is the case of $41 \%$ of all CME's, are associated with solar type II radio bursts and interplanetary shocks, while 
$30 \%$ of all solar type II radio bursts are accompanied neither by CME's nor by interplanetary shocks. Furthermore, some interplanetary shocks are not associated with solar type II radio bursts. Maxwell et al. (1985) reported on a CME event accompanied by a type II radio burst. They showed that the shock wave associated with the type II burst was piston-driven by the CME. On the other hand, Gopalswamy \& Kundu (1992) recently concluded by means of simultaneous measurements of type II radio bursts and CME's that solar type II radio bursts are generated by flare blast waves and not by CME's. A detailed knowledge of the properties of solar type II radio bursts and their associated shock waves is important for studying solar related events in the heliosphere as done by the ULYSSES satellite during the time interval under consideration. The URAP experiment aboard ULYSSES (Stone et al. 1992) is able to receive the radio radiation in the range $1 \mathrm{kHz}-1 \mathrm{MHz}$. According to the heliospheric density model (Bougeret et al. 1984) the $1 \mathrm{MHz}$ plasma frequency level corresponds to a distance of $0.1 \mathrm{AU}=21$ solar radii from the Sun. It should be noted, that the 40 $\mathrm{MHz}$ plasma frequency level, i.e., the lowest frequency of the observations presented, corresponds to a distance of 2 solar radii from the Sun (Mann et al. 1992).

In Sect. 2 we describe the data analysis of the observed solar type II radio bursts. The results of the statistical investigation of our data is given in Sect. 3. The paper is closed with some final remarks (Sect. 4).

\section{Data analysis}

Table 1 includes all solar type II radio bursts detected by the radiospectrograph of the Astrophysikalisches Institut Potsdam in Tremsdorf in the range $40-800 \mathrm{MHz}$ during the time period September 1, 1990 - December 31, 1993. Sixty five of the 100 solar type II radio bursts detected are analyzed to obtain their properties in the dynamic radio spectra, e.g., instantaneous bandwidth, drift rate etc.. These 65 type II bursts (cf. Table 2) are chosen because their spectral features are clearly visible and, consequently, their spectral properties can be determined from the observations.

Figure 2 shows a schematic sketch of the dynamic radio spectra of a solar type II radio burst. The instrument employed (Mann et al. 1992) records the radio intensity in terms of steps of a grey scale on a black-white film. The radio intensity is measured in arbitrary units. In the frequency range considered $(40-800 \mathrm{MHz})$ the background is largely nonuniform due to the instrumental noise, the galactic background, and the quiet Sun. The gauging of the instrument showed that a jump of the intensity of roughly $3 \mathrm{~dB}$ can just yet seen as a jump from one grey step to the next one. The measurements of bursts reported here are carried out as follows. The time is divided in $\mathrm{N}$ equidistant intervals of $10 \mathrm{~s}$, i.e., $t_{i+1}-t_{i}=10 \mathrm{~s}$, in each individual dynamic radio spectrum containing a type II radio burst. At each time $t_{i}$ the frequencies $f_{l, i}^{\mathrm{F}}, f_{u, i}^{\mathrm{F}}, f_{l, i}^{\mathrm{H}}$, and $f_{u, i}^{\mathrm{H}}$ are determined from the measurements (cf. Fig. 2 ) in such a way that the intensity is increasing above the background level by one grey scale step, i.e., an increase of approximately $3 \mathrm{~dB}$, at the threshold. In some cases of the type II bursts investigated the peak intensity exceeds the saturation level, so that the frequency at the peak intensity can not be determined. All parameters are derived from this data set.

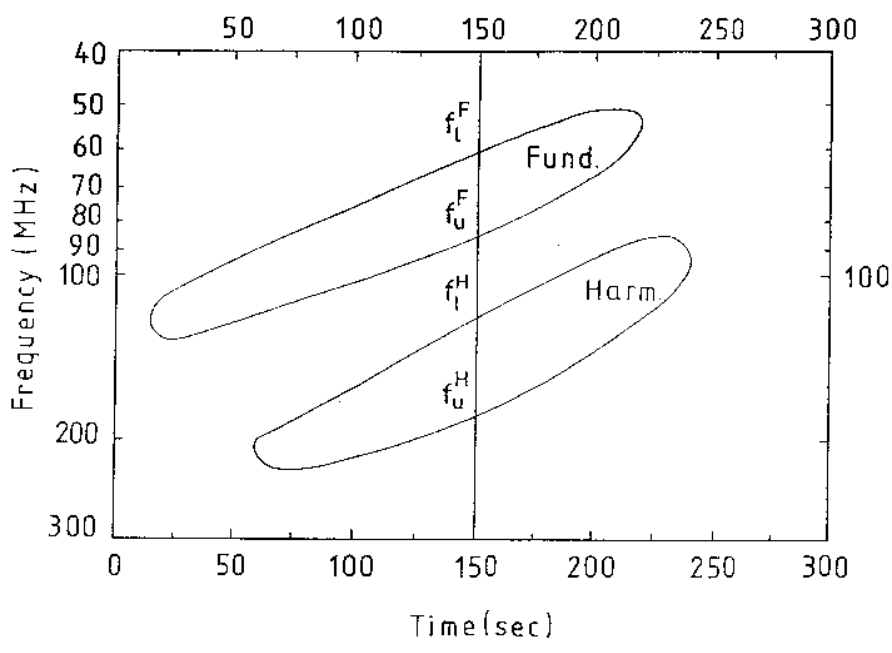

Fig. 2. Schematic sketch of the dynamic radio spectrum of a solar type II radio burst

The mean instantaneous width of the fundamental and harmonic band, i.e., $\left\langle(\Delta f / f)_{\mathrm{F}}\right\rangle$ and $\left\langle(\Delta f / f)_{\mathrm{H}}\right\rangle$, are calculated by

$$
\begin{aligned}
& \left\langle\left(\frac{\Delta f}{f}\right)_{\mathrm{F}}\right\rangle=\frac{1}{N} \sum_{i=1}^{N} \frac{f_{u, i}^{\mathrm{F}}-f_{l, i}^{\mathrm{F}}}{f_{l, i}^{\mathrm{F}}} \\
& \text { and } \\
& \left\langle\left(\frac{\Delta f}{f}\right)_{\mathrm{H}}\right\rangle=\frac{1}{N} \sum_{i=1}^{N} \frac{f_{u, i}^{\mathrm{H}}-f_{l, i}^{\mathrm{H}}}{f_{l, i}^{\mathrm{H}}}
\end{aligned}
$$

respectively. The linear regression analysis of the pairs $\left\{f_{l, i}^{\mathrm{F}}, t_{i}\right\},\left\{f_{u, i}^{\mathrm{F}}, t_{i}\right\},\left\{f_{l, i}^{\mathrm{H}}, t_{i}\right\}$ and $\left\{f_{u, i}^{\mathrm{H}}, t_{i}\right\}$ for all $i$ 's $(i$ $=1, \ldots, N)$ provides the linear functions $f_{k}^{\mathrm{F}}=a_{k}^{\mathrm{F}}+b_{k}^{\mathrm{F}} t$ and $f_{k}^{\mathrm{H}}=a_{k}^{\mathrm{H}}+b_{k}^{\mathrm{H}} t$ with $k=u, l$, respectively. Then, the drift rates of the fundamental and harmonic band, $D_{f, \mathrm{~F}}$ and $D_{f, \mathrm{H}}$, are determined by

$$
D_{f, \mathrm{~F}}=\frac{b_{l}^{\mathrm{F}}+b_{u}^{\mathrm{F}}}{2} \text { and } D_{f, \mathrm{H}}=\frac{b_{l}^{\mathrm{H}}+b_{u}^{\mathrm{H}}}{4}
$$

respectively. (On the other hand, the drift rate could also be derived from the frequencies at the peak intensity. But this method was not used since the peak intensity can not accurately be determined for all cases.) Generally, the 
Table 1. List of all solar type II radio bursts observed by the radiospectrograph of the Astrophysikalisches Institut Potsdam in Tremsdorf in the time period September 1, 1990 - December 31, 1993

\begin{tabular}{|c|c|c|c|c|}
\hline date & start & end & kind of event & freq. range \\
\hline 90.09 .14 & 1340.0 & 1419.3 & II, IIIG & $40-90$ \\
\hline 90.10 .01 & 1319.9 & 1327.3 & II, C, U, IIIB & $120-170$ \\
\hline 90.10 .10 & 0654.9 & 0658.8 & II, IIIG & $100-170$ \\
\hline 90.10 .15 & 1226.8 & 1239.0 & DCIM, II, C, IIIB & $50-800$ \\
\hline 90.12 .10 & 0749.0 & $0758 \mathrm{U}$ & II, IIIG & $100-170$ \\
\hline 90.12 .14 & $1333.9 \mathrm{E}$ & 1343.3 & II, IIIG & $60-450$ \\
\hline 90.12 .20 & $0722.8 \mathrm{U}$ & 0743.7 & II, IV, P, RS & $40-130$ \\
\hline 90.12 .23 & 0947.5 & 1000.0 & IV, IIIG, II & $40-400$ \\
\hline 90.12 .30 & $1315 \mathrm{U}$ & $1347 \mathrm{U}$ & IIIG, IV, II & $100-350$ \\
\hline 91.01 .08 & 1415.3 & 1416.5 & II? & $120-170$ \\
\hline 91.01 .18 & 1403.1 & 1421.6 & IV, II, HARM & $40-800$ \\
\hline 91.02 .01 & 1144.4 & 1157.0 & IIIG, IV, II & $40-90$ \\
\hline 91.02 .19 & 1230.8 & 1239.4 & II? & $50-90$ \\
\hline 91.02 .25 & 0812.3 & $0843.5 \mathrm{U}$ & IIIGG, RSG, IV, II & $40-400$ \\
\hline 91.03 .02 & 0735.2 & 0736.7 & UNCLF, II? & $120-150$ \\
\hline 91.03 .04 & 1359.5 & 1413.8 & UGG, II, H, HARM, IV & $50-800$ \\
\hline 91.03 .05 & 0940.9 & 0943.5 & II & $40-140$ \\
\hline 91.03 .07 & 0747.3 & $1500 \mathrm{U}$ & UGG, IV, II, HARM, IIIGG, P, I, DC & $40-800$ \\
\hline 91.03 .12 & 1237.5 & 1257.1 & U, IIIGG, IV, II, H, HARM, RS & $40-800$ \\
\hline 91.03 .16 & 1047.9 & 1105.7 & SPIKES, IIIGG, IV, II, C, Z & $40-800$ \\
\hline 91.03 .16 & 1239.1 & 1259.2 & IIIGG, IV, P, II?, SPIKES & $40-550$ \\
\hline 91.03 .22 & 0828.8 & $0850.3 \mathrm{U}$ & IIIGG, IV, II, H & $40-400$ \\
\hline 91.03 .22 & 1146.1 & 1203.5 & IIIGG, V, II?, IV & $40-400$ \\
\hline 91.03 .23 & $1227.0 \mathrm{U}$ & 1247.0 & IV $, P, Z, F, I I I G G_{1}$ II? & $40-800$ \\
\hline 91.03 .29 & 0641.8 & 0701.1 & SPIKES, IIIGG, U, IV, II, H, HARM & $40-800$ \\
\hline 91.04 .02 & 1001.6 & 1021.0 & IIIG, RS, UG, II, H, HARM, IV & $40-700$ \\
\hline 91.04 .11 & 1113.4 & 1142.5 & IIIGG, II, H, HARM, IV, P & $70-800$ \\
\hline 91.04 .20 & $0959 \mathrm{U}$ & $1023.0 \mathrm{U}$ & IV $, \mathrm{II}, \mathrm{H}, \mathrm{P}$ & $40-170$ \\
\hline 91.04 .29 & 1408.5 & 1416.0 & II & $100-170$ \\
\hline 91.05 .09 & $0717.0 \mathrm{U}$ & $0753 \mathrm{U}$ & II, $\mathrm{H}$ & $40-170$ \\
\hline 91.05 .16 & 0645.8 & $0737 \mathrm{U}$ & IIIGG, U, IV, II & $40-800$ \\
\hline 91.05 .30 & 0937.0 & 0940.7 & IIIB, RS, II, H, HARM & $150-800$ \\
\hline 91.06 .01 & 1502.9 & 1504.8 & II? & $200-400$ \\
\hline 91.06 .02 & $1412 \mathrm{U}$ & $1757 \mathrm{U}$ & IV, IIIG, II?, C, P, F, Z & $100-800$ \\
\hline 91.06 .07 & 0616.5 & $0623 \mathrm{U}$ & II & $40-90$ \\
\hline 91.06 .10 & $1356.6 \mathrm{U}$ & $1411.4 \mathrm{U}$ & IIIGG, II, HARM & $40-400$ \\
\hline 91.06 .15 & 0814.2 & $1146 \mathrm{U}$ & II, H, HARM, IV, UG, IIIGG, P & $40-800$ \\
\hline 91.06 .21 & 0836.7 & 0907.0 & IIIGG, RS, IV, II, H, HARM & $40-500$ \\
\hline 91.06 .21 & $1237 \mathrm{U}$ & $1252 \mathrm{U}$ & II? & $40-90$ \\
\hline 91.06 .28 & 0800.7 & 0813.0 & U, IIIGG, IV, II, H, HARM & $40-300$ \\
\hline 91.06 .28 & 1428.9 & 1435.8 & IIIGG, IV, II, HARM, RSG & $40-600$ \\
\hline 91.07 .05 & 1002.8 & 1012.6 & IIIGG, RS, IV, II, H & $40-400$ \\
\hline 91.07 .10 & $1203 \mathrm{U}$ & $1531 \mathrm{U}$ & IIIGG, IV, II, U, I, F, Z & $40-400$ \\
\hline 91.07 .11 & 1036.3 & 1042.0 & II & $40-70$ \\
\hline 91.07 .17 & $0627 \mathrm{E}$ & $0652 U$ & IIIGG, IV, H, H, HARM, RS & $40-800$ \\
\hline 91.07 .22 & 0946.0 & $1456 \mathrm{U}$ & IIIG, IV, U, II, H, HARM, P, RS & $40-800$ \\
\hline 91.09 .02 & 1000.9 & 1005.5 & II & $40-60$ \\
\hline 91.09 .08 & 0912.8 & 1015 & IIIG, V, II, H, HARM, IV & $40-350$ \\
\hline 91.09 .24 & 0750.5 & $0923 \mathrm{U}$ & IV $, \mathrm{H}, \mathrm{U}, \mathrm{LIIGG}$ & $60-800$ \\
\hline 91.10 .05 & 0757.7 & $0823 \mathrm{U}$ & IIIG, IV, II, HARM & $40-800$ \\
\hline
\end{tabular}

\begin{tabular}{|c|c|c|c|c|}
\hline date & start & end & kind of event & freq. range \\
\hline 91.10 .30 & $0628 \mathrm{E}$ & $0820 \mathrm{U}$ & II, IV & $100-170$ \\
\hline 91.12 .26 & $0759.6 \mathrm{U}$ & $0906.0 \mathrm{U}$ & II?, IV, IIIGG & $40-400$ \\
\hline 91.12 .26 & 0932.0 & 1014.2 & IIIGG, IV, II, U, P & $40-800$ \\
\hline 91.12 .27 & $1106.7 \mathrm{E}$ & 1125.5 & IV, II, HARM, IIIG & $40-800$ \\
\hline 91.12 .28 & 1226.4 & 1227.3 & II? & $250-400$ \\
\hline 91.12 .30 & $1013 \mathrm{U}$ & 1035.3 & IIIG, IV, II, HARM, SPIKES & $40-800$ \\
\hline 92.01 .09 & 1240.3 & 1245.3 & II, IV & $100-400$ \\
\hline 92.02 .02 & 0857.6 & $0951 \mathrm{U}$ & IIIGG, IV, II, H, P & $50-800$ \\
\hline 92.02 .07 & 1144.4 & $1319 \mathrm{U}$ & IIIG, IV, U, II? & $40-800$ \\
\hline 92.02 .27 & 0951.2 & $1045 \mathrm{U}$ & IIG, H, HARM, IV, P, IIIG, Z, F, UG & $40-800$ \\
\hline 92.03 .09 & 1336.4 & 1354.5 & II, H, IV , F, IIIGG, RS & $120-350$ \\
\hline 92.03 .11 & 0905.9 & 0906.4 & II?, (UNCLF) & $100-300$ \\
\hline 92.04 .30 & 0808.0 & 0809.4 & II? & $40-50$ \\
\hline 92.05 .07 & 0643.6 & $0654.5 \mathrm{U}$ & $\mathrm{II}, \mathrm{H}, \mathrm{C}$ & $40-90$ \\
\hline 92.06 .08 & 0828.5 & $1115 \mathrm{U}$ & II, HARM, IV, I, F, P, Z, RS, IIIGG & $40-800$ \\
\hline 92.06 .09 & $0605 \mathrm{E}$ & $0639 \mathrm{U}$ & IIIG, IV, II, I, P, U? & $100-800$ \\
\hline 92.06 .16 & 0925.1 & 0927.5 & II? & $70-130$ \\
\hline 92.07 .16 & 0727.0 & 07310 & $(\mathrm{II}, \mathrm{H}) ?$ & $200-400$ \\
\hline 92.08 .03 & 0831.8 & $0837.0 \mathrm{U}$ & II? & $200-400$ \\
\hline 92.07 .14 & 1016.1 & 1040.7 & IIIGG, RS, IV, P, II, H, HARM & $40-400$ \\
\hline 92.08 .10 & 1127.8 & 1128.5 & II? & $300-500$ \\
\hline 92.09 .03 & 0930.8 & 0932.2 & II & $100-150$ \\
\hline 92.09 .11 & 0604.6 & $0615 \mathrm{U}$ & IIIGG, IV, II, H, HARM & $50-500$ \\
\hline 92.10 .01 & 1039.1 & $1103 \mathrm{U}$ & IIIGG, II, H, HARM & $40-400$ \\
\hline 92.10 .06 & 0950.1 & $1007.5 \mathrm{U}$ & IIIG, II? & $40-170$ \\
\hline 92.10 .06 & 1043.9 & 1046.0 & IIIG, V, II? & $40-400$ \\
\hline 92.10 .20 & 0909.5 & $0928 \mathrm{U}$ & IIIGG, IV, II, H, HARM, RSG & $40-800$ \\
\hline 92.10 .28 & $1007.9 \mathrm{U}$ & $1155 \mathrm{U}$ & IIIGG, IV, II, H, HARM, RS, P & $40-800$ \\
\hline 92.11 .09 & 1114.6 & 1120.5 & IIIGG, (IV, II $) ?$ & $40-170$ \\
\hline 92.12 .09 & 1117.8 & 1125.2 & II, H, HARM, IIIGG & $100-600$ \\
\hline 92.12 .30 & 0818.8 & $0832 \mathrm{U}$ & IIIGG, IV, II, RS, SPIKES & $40-300$ \\
\hline 92.12 .30 & 1031.8 & $1040 \mathrm{U}$ & II, HARM, C & $40-90$ \\
\hline 93.01 .01 & 1150.6 & 1158.0 & IIIGG, IV, II, H, HARM, RS, SPIKES & $40-500$ \\
\hline 93.01 .01 & 1335.6 & 1353.0 & IIIGG, RS, IV, II, H, U & $40-400$ \\
\hline 93.01 .07 & 0828.1 & $1005 \mathrm{U}$ & (II, HARM)?, IV, DCIM, P & $60-800$ \\
\hline 93.01 .15 & 0923.2 & $0930.6 \mathrm{U}$ & IV, II, H, U? & $100-500$ \\
\hline 93.02 .18 & 0933.4 & 1019.6 & II?, IIIGG, I, RS, C & $40-400$ \\
\hline 93.02 .18 & 1048.3 & $1111 \mathrm{U}$ & IIIG, IV, II, H, HARM & $40-400$ \\
\hline 93.03 .04 & 1213.8 & 1311.9 & IIIGG, IV, II?, U & $40-500$ \\
\hline 93.03 .19 & 1242.4 & $1321.0 \mathrm{U}$ & II?, IIIGG, RS & $40-400$ \\
\hline 93.03 .29 & 1019.2 & 1046.4 & IIIGG, RS, II? & $40-700$ \\
\hline 93.03 .29 & 1251.3 & 1258.1 & IV, IIIGG, U, II? & $80-700$ \\
\hline 93.04 .30 & 1427 & 1432 & II & $40-70$ \\
\hline 93.05 .25 & 1513.0 & 1516.3 & II & $40-90$ \\
\hline 93.05 .26 & 1029.2 & 1033.8 & II?, I, IIIGG, RS & $40-600$ \\
\hline 93.06 .07 & 1410.4 & $1529 \mathrm{U}$ & HIGG, IV, II, H, HARM, Z & $40-170$ \\
\hline 93.07 .22 & 0852.2 & 0915.8 & II, H, HARM & $40-150$ \\
\hline 93.09 .27 & 1207.1 & $1219 \mathrm{U}$ & IIIG, II, H, HARM, P, DCIM & $40-800$ \\
\hline 93.12 .27 & 0911.6 & 0918.1 & IIIG, II, HARM & $110-500$ \\
\hline 93.12 .28 & 1208.1 & 1218.5 & IIIG, II, H, HARM & $40-700$ \\
\hline
\end{tabular}

Abbreviations at times: E, early as given; U, later than given event remarks: I, II, III, IV, V; type I, II, III, IV, V burst; UNCLF, unclassified events; B, single burst; S, storm bursts; U, type U bursts; G small group of bursts (<10); GG, large group of bursts (> 10); C any continuum underlying other events; F, fluctuating continuum; RS, reverse drift bursts; H, herringbones; P, pulsations; Z, zebra bursts; HARM, harmonic emission; SPIKES, spike bursts; DCIM, events in the decimetric range

originally measured drift rates of the harmonic bands are roughly twice of those of the fundamental bands. In order to compare both the originally measured drift rate of the harmonic band is divided by 2 as done in Eq. (2) as well as in Table 2 and Sect. 3. Finally, the mean value of the ratio $\left\langle f_{\mathrm{H}} / f_{\mathrm{F}}\right\rangle$ for the middle frequencies of the harmonic and the fundamental bands is found by

$$
\left\langle\frac{f_{\mathrm{H}}}{f_{\mathrm{F}}}\right\rangle=\frac{1}{N} \sum_{i=1}^{N} \frac{f_{l, i}^{\mathrm{H}}+f_{u, i}^{\mathrm{H}}}{f_{l, i}^{\mathrm{F}}+f_{u, i}^{\mathrm{F}}}
$$

In Table 2 the mean values and the standard deviation of the instantaneous bandwidths $\Delta f / f$, the drift rate
$D_{f}$, and the ratio $f_{\mathrm{H}} / f_{\mathrm{F}}$ are listed for all type II bursts analyzed. It should be mentioned that the instantaneous bandwidth and the drift rate are separately determined for the fundamental and harmonic bands as given in Table 2 .

The method of determining the frequencies $f_{l, i}^{\mathrm{F}}, f_{u, i}^{\mathrm{F}}$, $f_{l, i}^{\mathrm{H}}$, and $f_{u, i}^{\mathrm{H}}$ leads to some errors in contrast to the deterimation at the $3 \mathrm{~dB}$ increase of the intensity. But this error can be considered to be small since our method is roughly the same. Furthermore, the calculation of the instantaneous bandwidth (cf. Eq. (1)), the drift rate (cf. Eq. (2)) and the ratio $f_{\mathrm{H}} / f_{\mathrm{F}}$ (cf. Eq. $\left.(3)\right)$ is done by a statistical method, so that the above mentioned errors are reduced by this averaging. 
Table 2. List of the spectral characteristics (general spectral structure (fundamental (F), harmonic $(\mathrm{H})$ ), instantaneous bandwidth $\langle\Delta f / f\rangle$ and drift rate $\left\langle D_{f}\right\rangle$ of the fundamental (left) and harmonic (right) band, respectively, as well as ratio $\left\langle f_{\mathrm{H}} / f_{\mathrm{F}}\right\rangle$ ) of the solar type II radio bursts investigated (cf. Table 1). The mean values and the standard deviations are given in the last panel

\begin{tabular}{|c|c|c|c|c|c|c|c|}
\hline no. & $\begin{array}{c}\text { date } \\
\text { no. of day }\end{array}$ & $\begin{array}{l}\text { universal } \\
\text { time }\end{array}$ & structure & $\left\langle\frac{\Delta f}{f}\right\rangle$ & $\begin{array}{c}\left\langle D_{\mathrm{f}}\right\rangle \\
{[\mathrm{MHz} / \mathrm{s}]}\end{array}$ & $\begin{array}{c}\text { freq. range } \\
{[\mathrm{MHz}]}\end{array}$ & $\left\langle\frac{f_{\mathrm{H}}}{f_{\mathrm{F}}}\right\rangle$ \\
\hline 1 & $\begin{array}{c}90.09 .14 \\
257 \\
\end{array}$ & $1341-1349$ & $\begin{array}{c}\text { F } \\
\text { IIIGG, II }\end{array}$ & $0.27 \pm 0.05$ & $-0.063 \pm 0.002$ & $66-50$ & \\
\hline 2 & $\begin{array}{l}90.10 .01 \\
274\end{array}$ & $1325-1328$ & $\begin{array}{c}\text { F } \\
\text { IIIB, II }\end{array}$ & $0.29 \pm 0.06$ & $-0.048 \pm 0.023$ & $63-55$ & - \\
\hline 3 & $\begin{array}{c}90.10 .10 \\
283\end{array}$ & $0657-0659$ & $\begin{array}{c}\text { F? } \\
\text { IIIG, II }\end{array}$ & $0.19 \pm 0.03$ & $-0.23 \pm 0.01$ & $145-110$ & - \\
\hline 4 & $\begin{array}{c}90.10 .15 \\
288\end{array}$ & $1229-1231$ & $\begin{array}{c}\text { F? } \\
\text { II, IIIB }\end{array}$ & $0.27 \pm 0.05$ & $-0.25 \pm 0.02$ & $143-103$ & 一 \\
\hline 5 & $\begin{array}{c}90.12 .10 \\
344\end{array}$ & $0749-0803$ & $\begin{array}{c}\text { F, H } \\
\text { IIIG, II, IIIG }\end{array}$ & $\begin{array}{c}0.53 \pm 0.13 \\
-\mid 0.53\end{array}$ & $\begin{array}{r}-0.10 \pm 0.04 \\
-\mid-0.10\end{array}$ & $81-45$ & $\begin{array}{c}2.03 \pm 0.07 \\
-\mid 2.03\end{array}$ \\
\hline 6 & $\begin{array}{c}90.12 .14 \\
348 \\
\end{array}$ & $1335-1343$ & $\begin{array}{c}\text { F, H } \\
\text { IIIG, II }\end{array}$ & $\begin{array}{c}0.30 \pm 0.12 \\
0.20 \mid 0.43 \\
\end{array}$ & $\begin{array}{l}-0.28 \pm 0.11 \\
-0.34 \mid-0.22\end{array}$ & $125-110$ & $\begin{array}{r}1.94 \pm 0.07 \\
-11.94 \\
\end{array}$ \\
\hline 7 & $\begin{array}{c}90.12 .20 \\
354 \\
\end{array}$ & $0725-0730$ & $\begin{array}{c}\mathrm{F}, \mathrm{H} \\
\mathrm{RS}, \mathrm{II}, \mathrm{IV}\end{array}$ & $\begin{array}{c}0.16 \pm 0.04 \\
0.14 \mid 0.18\end{array}$ & $\begin{array}{l}-0.07 \pm 0.04 \\
-0.08 \mid-0.07\end{array}$ & $53-41$ & $\begin{array}{l}1.99 \pm 0.13 \\
1.87 \mid 2.11 \\
\end{array}$ \\
\hline 8 & $\begin{array}{l}90.12 .23 \\
357\end{array}$ & 0949-0957 & $\begin{array}{c}\mathbf{F}, \mathrm{H} \\
\text { IV }, \text { IIIGG, II }\end{array}$ & $\begin{array}{c}0.36 \pm 0.16 \\
0.38 \mid 0.36\end{array}$ & $\begin{array}{l}-0.18 \pm 0.05 \\
-0.22 \mid-0.15\end{array}$ & $122-43$ & $\begin{array}{c}1.97 \pm 0.23 \\
1.85 \mid 2.06 \\
\end{array}$ \\
\hline 9 & $\begin{array}{c}90.12 .30 \\
364\end{array}$ & $1321-1324$ & $\begin{array}{c}\text { H } \\
\text { IIIG, IV, II }\end{array}$ & $\begin{array}{c}0.35 \pm 0.02 \\
-\mid 0.35 \\
\end{array}$ & $\begin{array}{r}-0.23 \pm 0.06 \\
-\mid-0.23\end{array}$ & $\approx 140$ & - \\
\hline 10 & $\begin{array}{c}91.01 .18 \\
18\end{array}$ & $1411-1421$ & $\begin{array}{c}\text { F? } \\
\text { VI, II }\end{array}$ & $0.12 \pm 0.04$ & $-0.19 \pm 0.07$ & $156-52$ & 一 \\
\hline 11 & $\begin{array}{c}91.02 .01 \\
32 \\
\end{array}$ & $1451-1456$ & $\begin{array}{c}\mathrm{F} \\
\mathrm{IIIG}, \mathrm{IV}, \mathrm{II}\end{array}$ & $0.13 \pm 0.04$ & $-0.11 \pm 0.01$ & $82-55$ & - \\
\hline 12 & $\begin{array}{c}91.02 .25 \\
56\end{array}$ & $0834-0838$ & $\begin{array}{c}F \\
\text { IIIGG, IV, II }\end{array}$ & $0.30 \pm 0.05$ & $-0.07 \pm 0.03$ & $60-42$ & - \\
\hline 13 & $\begin{array}{c}91.03 .04 \\
63\end{array}$ & $1402-1405$ & $\begin{array}{c}F, H \\
\text { UGG, IV, II }\end{array}$ & $\begin{array}{c}0.48 \pm 0.39 \\
0.20 \mid 0.75\end{array}$ & $\begin{array}{l}-0.26 \pm 0.02 \\
-0.28 \mid-0.25\end{array}$ & $133-103$ & $\begin{array}{l}1.81 \pm 0.40 \\
2.09 \mid 1.52\end{array}$ \\
\hline 14 & $\begin{array}{c}91.03 .05 \\
64\end{array}$ & 0941-0943 & $\begin{array}{c}\mathrm{F}, \mathrm{H} \\
\text { II }\end{array}$ & $\begin{array}{c}0.16 \pm 0.05 \\
-\mid 0.16\end{array}$ & $\begin{array}{r}-0.10 \pm 0.02 \\
-\mid-0.10\end{array}$ & $\approx 90-60$ & - \\
\hline 15 & $\begin{array}{c}91.03 .07 \\
66 \\
\end{array}$ & $0756-0758$ & $\begin{array}{c}\text { F, H } \\
\text { IV, II, IIIGG }\end{array}$ & $\begin{array}{c}0.23 \pm 0.08 \\
0.17 \mid 0.28 \\
\end{array}$ & $\begin{array}{l}-0.09 \pm 0.08 \\
-0.08 \mid-0.10\end{array}$ & $53-40$ & $\begin{array}{c}2.06 \pm 0.05 \\
2.10 \mid 2.03 \\
\end{array}$ \\
\hline 16 & $\begin{array}{c}91.03 .12 \\
71 \\
\end{array}$ & $1252-1257$ & $\begin{array}{c}\text { F, H } \\
\text { IIIGG, IV, II }\end{array}$ & $\begin{array}{c}0.39 \pm 0.10 \\
0.39 \mid 0.31 \\
\end{array}$ & $\begin{array}{l}-0.08 \pm 0.01 \\
-0.10 /-0.06\end{array}$ & $62-46$ & $\begin{array}{c}2.08 \pm 0.08 \\
2.05 \mid 2.12 \\
\end{array}$ \\
\hline 17 & $\begin{array}{c}91.03 .22 \\
81 \\
\end{array}$ & 0844-0852 & $\begin{array}{c}\text { F? } \\
\text { IIIGG, IV, II }\end{array}$ & $\begin{array}{l}0.25 \pm 0.12 \\
0.25 \mid-\end{array}$ & $\begin{array}{l}-0.13 \pm 0.03 \\
-0.13 \mid-\end{array}$ & $118-63$ & - \\
\hline 18 & $\begin{array}{c}91.03 .23 \\
82 \\
\end{array}$ & $1237-1239$ & $\begin{array}{c}\text { H? } \\
\text { IV, IIIGG }\end{array}$ & $\begin{array}{c}0.11 \pm 0.03 \\
-\mid 0.11 \\
\end{array}$ & $\begin{array}{r}-0.19 \pm 0.03 \\
-\mid-0.19\end{array}$ & $160-150$ & - \\
\hline 19 & $\begin{array}{c}91.03 .29 \\
88\end{array}$ & $0649-0657$ & $\begin{array}{c}\text { F, H } \\
\text { IV, II, IIIGG }\end{array}$ & $\begin{array}{c}0.52 \pm 0.26 \\
0.26 \mid 0.78\end{array}$ & $\begin{array}{l}-0.12 \pm 0.02 \\
-0.10 \mid-0.14\end{array}$ & $\approx 100-49$ & $\begin{array}{l}1.80 \pm 0.59 \\
2.22 \mid 1.38\end{array}$ \\
\hline 20 & $\begin{array}{c}91.04 .02 \\
92 \\
\end{array}$ & $1007-1009$ & $\begin{array}{c}\text { F, H } \\
\text { IIIG, II, IV }\end{array}$ & $\begin{array}{c}0.47 \pm 0.20 \\
0.33 \mid 0.61 \\
\end{array}$ & $\begin{array}{l}-0.14 \pm 0.03 \\
-0.17 \mid-0.11 \\
\end{array}$ & $68-50$ & $\begin{array}{l}2.10 \pm 0.07 \\
2.10 \mid-\end{array}$ \\
\hline 21 & $\begin{array}{c}91.04 .11 \\
101 \\
\end{array}$ & $1116-1118$ & $\begin{array}{c}\text { F, H } \\
\text { IIIGG, II, IV }\end{array}$ & $\begin{array}{c}0.32 \pm 0.21 \\
0.17 \mid 0.46 \\
\end{array}$ & $\begin{array}{l}-0.36 \pm 0.02 \\
-0.37 \mid-0.34\end{array}$ & $149-101$ & $\begin{array}{c}1.82 \pm 0.30 \\
2.03 \mid 1.61 \\
\end{array}$ \\
\hline \multirow[t]{2}{*}{22} & $\begin{array}{c}91.04 .20 \\
110\end{array}$ & $1007-1008$ & $\begin{array}{c}\text { F? } \\
\text { IV }, \text { II }\end{array}$ & - & $-0.33 \pm 0.10$ & $80-43$ & - \\
\hline & & & & fund. harm. & fund. / harm. & & \begin{tabular}{l|l} 
upper & lower \\
\end{tabular} \\
\hline
\end{tabular}


Table 2. continued

\begin{tabular}{|c|c|c|c|c|c|c|c|}
\hline no. & $\begin{array}{c}\text { date } \\
\text { no. of day }\end{array}$ & $\begin{array}{l}\text { universal } \\
\text { time }\end{array}$ & structure & $\left\langle\frac{\Delta f}{f}\right\rangle$ & $\begin{array}{c}\left\langle D_{\mathrm{f}}\right\rangle \\
{[\mathrm{MHz} / \mathrm{s}]} \\
\end{array}$ & $\begin{array}{c}\text { freq. range } \\
{[\mathrm{MHz}]}\end{array}$ & $\left\langle\frac{f_{\mathrm{H}}}{f_{\mathrm{F}}}\right\rangle$ \\
\hline 23 & $\begin{array}{c}91.04 .29 \\
119\end{array}$ & $1409-1411$ & $\begin{array}{l}\text { F? } \\
\text { II }\end{array}$ & $0.29 \pm 0.04$ & $-0.12 \pm 0.06$ & $120-107$ & - \\
\hline 24 & $\begin{array}{c}91.05 .09 \\
129\end{array}$ & $0717-0721$ & $\begin{array}{l}\text { F? } \\
\text { II }\end{array}$ & $0.20 \pm 0.03$ & $-0.08 \pm 0.01$ & $129-108$ & - \\
\hline 25 & $\begin{array}{c}91.05 .16 \\
136\end{array}$ & $0708-0712$ & $\begin{array}{c}\mathrm{F} \\
\text { IIIGG, IV, II }\end{array}$ & $0.18 \pm 0.07$ & $-0.01 \pm 0.004$ & $55-49$ & - \\
\hline 26 & $\begin{array}{c}91.05 .30 \\
150\end{array}$ & $0939-0941$ & $\begin{array}{c}\mathrm{F}, \mathrm{H} \\
\text { IIIB, II }\end{array}$ & $\begin{array}{c}0.31 \pm 0.10 \\
-\mid 0.31\end{array}$ & $\begin{array}{l}-0.48 \pm 0.07 \\
-0.41 \mid-0.55\end{array}$ & $225-198$ & $\begin{array}{l}2.01 \pm 0.06 \\
2.01 \mid-\end{array}$ \\
\hline 27 & $\begin{array}{c}91.06 .02 \\
153\end{array}$ & $1416-1418$ & $\begin{array}{c}\mathrm{F} ? \\
\text { IV, IIIG, II }\end{array}$ & $0.16 \pm 0.02$ & $-0.13 \pm 0.01$ & $123-109$ & - \\
\hline 28 & $\begin{array}{c}91.06 .07 \\
158\end{array}$ & $0617-0618$ & $\begin{array}{l}\text { F? } \\
\text { II }\end{array}$ & $0.59 \pm 0.07$ & $-0.09 \pm 0.05$ & $52-45$ & - \\
\hline 29 & $\begin{array}{c}91.06 .10 \\
161\end{array}$ & $1401-1407$ & $\begin{array}{c}\mathrm{F}, \mathrm{H} \\
\mathrm{IIIGG}, \mathrm{II}\end{array}$ & $\begin{array}{c}0.38 \pm 0.10 \\
0.36 \mid 0.43 \\
\end{array}$ & $\begin{array}{l}-0.08 \pm 0.02 \\
-0.10 \dagger-0.06\end{array}$ & $74-47$ & $\begin{array}{c}2.07 \pm 0.10 \\
2.08 \mid 2.07 \\
\end{array}$ \\
\hline 30 & $\begin{array}{c}91.06 .15 \\
166\end{array}$ & $0816-0821$ & $\begin{array}{c}F, H \\
\text { II, IV, IIIGG }\end{array}$ & - & $\begin{array}{l}-0.37 \pm 0.03 \\
-0.35 \mid-0.39 \\
\end{array}$ & $83-41$ & - \\
\hline 31 & $\begin{array}{c}91.06 .21 \\
172 \\
\end{array}$ & $1237-1241$ & $\begin{array}{l}\mathrm{F} \\
\mathrm{II} \\
\end{array}$ & $0.12 \pm 0.03$ & $-0.04 \pm 0.02$ & $55-49$ & - \\
\hline 32 & $\begin{array}{c}91.06 .21 \\
172\end{array}$ & $0849-0851$ & $\begin{array}{c}\mathrm{F} \\
\mathrm{IIIGG}, \mathrm{IV}, \mathrm{II}\end{array}$ & $0.30 \pm 0.12$ & $-0.11 \pm 0.08$ & $55-42$ & - \\
\hline 33 & $\begin{array}{c}91.06 .28 \\
179 \\
\end{array}$ & $0809-0811$ & $\begin{array}{c}\mathrm{F}, \mathrm{H} \\
\text { IIIGG, IV, II }\end{array}$ & $\begin{array}{l}0.45 \pm 0.10 \\
0.45 \mid-\end{array}$ & $\begin{array}{l}-0.13 \pm 0.01 \\
-0.13:-0.12 \\
\end{array}$ & $66-40$ & - \\
\hline 34 & $\begin{array}{c}91.07 .05 \\
186 \\
\end{array}$ & $1008-1009$ & $\begin{array}{c}\text { F? } \\
\text { IIIGG, IV, II }\end{array}$ & $0.25 \pm 0.03$ & $-0.15 \pm 0.01$ & $121-108$ & - \\
\hline 35 & $\begin{array}{c}91.07 .10 \\
191\end{array}$ & $1218-1221$ & $\begin{array}{c}\text { F? } \\
\text { IIIGG, IV, II }\end{array}$ & $0.07 \pm 0.02$ & $-0.14 \pm 0.01$ & $137-107$ & - \\
\hline 36 & $\begin{array}{c}91.07 .11 \\
192 \\
\end{array}$ & $1036-1042$ & $\begin{array}{l}\mathrm{F} \\
\mathrm{II} \\
\end{array}$ & $0.27 \pm 0.08$ & $-0.04 \pm 0.02$ & $54-46$ & - \\
\hline 37 & $\begin{array}{c}91.07 .17 \\
198\end{array}$ & $0639-0651$ & $\begin{array}{c}\mathrm{H} \\
\text { IIIGG, IV, II }\end{array}$ & $0.33 \pm 0.05$ & $-0.022 \pm 0.001$ & 40 & - \\
\hline 38 & $\begin{array}{c}91.07 .22 \\
203\end{array}$ & $1005-1014$ & $\begin{array}{c}\text { F , H } \\
\text { IIIG, IV, U, II }\end{array}$ & $\begin{array}{c}0.33 \pm 0.04 \\
0.33 \mid 0.33\end{array}$ & $\begin{array}{l}-0.06 \pm 0.06 \\
-0.06 \mid-0.07\end{array}$ & $63-44$ & $\begin{array}{c}2.16 \pm 0.08 \\
-12.16\end{array}$ \\
\hline 39 & $\begin{array}{c}91.09 .02 \\
245\end{array}$ & $1001-1005$ & \begin{tabular}{|l|}
$\mathrm{F}$ \\
$\mathrm{II}$ \\
\end{tabular} & $0.17 \pm 0.05$ & $-0.04 \pm 0.02$ & $49-42$ & - \\
\hline 40 & $\begin{array}{c}91.09 .08 \\
251\end{array}$ & 0917-0927 & $\begin{array}{c}\text { F, H } \\
\text { IIIG, V, II, IV }\end{array}$ & $\begin{array}{c}0.38 \pm 0.07 \\
0.33 \mid 0.42 \\
\end{array}$ & $\begin{array}{l}-0.10 \pm 0.04 \\
-0.12 \mid-0.08 \\
\end{array}$ & $80-50$ & $\begin{array}{c}2.05 \pm 0.21 \\
1.90 \mid 2.13\end{array}$ \\
\hline 41 & $\begin{array}{c}91.09 .24 \\
267\end{array}$ & $0752-0800$ & $\begin{array}{c}\text { H? } \\
\text { IV, II, IIIGG }\end{array}$ & $0.41 \pm 0.08$ & $-0.18 \pm 0.1$ & I $33-52$ & - \\
\hline 42 & $\begin{array}{c}91.12 .26 \\
360 \\
\end{array}$ & $0949-0955$ & $\begin{array}{c}\text { F } \\
\text { IIIGG, IV, II }\end{array}$ & $0.32 \pm 0.04$ & $-0.049 \pm 0.004$ & $67-54$ & - \\
\hline 43 & $\begin{array}{c}91.12 .27 \\
361 \\
\end{array}$ & $1114-1117$ & $\begin{array}{c}\text { F, H } \\
\text { IV }, \text { II, IIIG }\end{array}$ & $\begin{array}{c}0.31 \pm 0.06 \\
-\mid 0.31 \\
\end{array}$ & $\begin{array}{l}-0.05 \pm 0.03 \\
-0.05 \mid-0.05\end{array}$ & $58-51$ & $\begin{array}{c}2.09 \pm 0.06 \\
2.05 \mid 2.13 \\
\end{array}$ \\
\hline \multirow[t]{2}{*}{44} & $\begin{array}{c}91.12 .30 \\
364\end{array}$ & $1022-1031$ & $\begin{array}{c}\text { F? } \\
\text { IIIG, IV, II }\end{array}$ & $-\ldots$ & $-0.09 \pm 0.03$ & $81-51$ & - \\
\hline & & & & fund. I harm. & fund. | harm. & & upper | lower \\
\hline
\end{tabular}


Table 2. continued

\begin{tabular}{|c|c|c|c|c|c|c|c|}
\hline no. & $\begin{array}{c}\text { date } \\
\text { no. of day }\end{array}$ & $\begin{array}{l}\text { universal } \\
\text { time }\end{array}$ & structure & $\left\langle\frac{\Delta f}{f}\right\rangle$ & $\begin{array}{c}\left\langle D_{\mathrm{f}}\right\rangle \\
{[\mathrm{MHz} / \mathrm{s}]}\end{array}$ & $\begin{array}{c}\text { freq. range } \\
{[\mathrm{MHz}]}\end{array}$ & $\left\langle\frac{f_{\mathrm{H}}}{f_{\mathrm{F}}}\right\rangle$ \\
\hline 45 & $\begin{array}{c}92.02 .02 \\
33 \\
\end{array}$ & $0910-0915$ & $\begin{array}{c}\mathrm{H} \\
\text { IIIGG, IV, II }\end{array}$ & - & $-0.07 \pm 0.03$ & $\approx 50$ & - \\
\hline 46 & $\begin{array}{c}92.02 .27 \\
58 \\
\end{array}$ & $0951-0954$ & $\mathrm{~F}, \mathrm{H}$ & $\begin{array}{c}0.48 \pm 0.40 \\
0.19 \mid 0.76\end{array}$ & $\begin{array}{l}-0.32 \pm 0.08 \\
-0.24 \mid-0.39\end{array}$ & $113-56$ & $\begin{array}{c}1.88 \pm 0.09 \\
1.94 \mid 1.81\end{array}$ \\
\hline 47 & $\begin{array}{c}92.05 .07 \\
128 \\
\end{array}$ & $0643-0651$ & $\begin{array}{l}\mathrm{F} \\
\mathrm{II}\end{array}$ & $0.27 \pm 0.02$ & $-0.043 \pm 0.022$ & $58-44$ & - \\
\hline 48 & $\begin{array}{c}92.07 .14 \\
196\end{array}$ & $1025-1029$ & $\begin{array}{c}\text { F? } \\
\mathrm{IIG}, \mathrm{IV}, \mathrm{IIIG}\end{array}$ & - & $-0.27 \pm 0.13$ & $\approx 100$ & - \\
\hline 49 & $\begin{array}{c}92.08 .03 \\
216 \\
\end{array}$ & $0832-0835$ & $\begin{array}{c}\mathrm{H} ? \\
\mathrm{II}\end{array}$ & $0.37 \pm 0.05$ & $-0.22 \pm 0.06$ & $155-125$ & 一 \\
\hline 50 & $\begin{array}{c}92.09 .03 \\
247 \\
\end{array}$ & 0931-0932 & $\begin{array}{l}\text { F? } \\
\text { II }\end{array}$ & - & $-0.21 \pm 0.05$ & $113-110$ & - \\
\hline 51 & $\begin{array}{c}92.09 .11 \\
255\end{array}$ & $0607-0610$ & $\begin{array}{c}\text { F? } \\
\text { IIIGG, IV, II }\end{array}$ & $0.23 \pm 0.05$ & $-0.39 \pm 0.08$ & $160-120$ & - \\
\hline 52 & $\begin{array}{c}92.10 .01 \\
275 \\
\end{array}$ & $1048-1052$ & $\begin{array}{c}\text { F, H } \\
\text { IIIGG, II }\end{array}$ & $\begin{array}{c}0.38 \pm 0.10 \\
0.39 \mid 0.30 \\
\end{array}$ & $\begin{array}{l}-0.07 \pm 0.04 \\
-0.08 \mid-0.06 \\
\end{array}$ & $50-42$ & $\begin{array}{c}2.17 \pm 0.13 \\
-\mid 2.17\end{array}$ \\
\hline 53 & $\begin{array}{c}92.10 .20 \\
294 \\
\end{array}$ & $0915-0924$ & $\begin{array}{c}\text { F, H } \\
\text { IIIGG, IV, II }\end{array}$ & $\begin{array}{c}0.74 \pm 0.16 \\
-\mid 0.74 \\
\end{array}$ & $\begin{array}{l}-0.13 \pm 0.06 \\
-0.18 \\
\end{array}$ & $47-41$ & $\begin{array}{l}2.20 \pm 0.10 \\
2.20 \mid-\end{array}$ \\
\hline 54 & $\begin{array}{c}92.10 .28 \\
302\end{array}$ & $1011-1021$ & $\begin{array}{c}\text { F, H } \\
\text { IIIGG, IV, II }\end{array}$ & $\begin{array}{c}0.25 \pm 0.02 \\
0.23 \mid 0.26\end{array}$ & $\begin{array}{l}-0.07 \pm 0.02 \\
-0.09 \mid-0.05\end{array}$ & $54-42$ & $\begin{array}{l}2.06 \pm 0.09 \\
2.061-\end{array}$ \\
\hline 55 & $\begin{array}{c}92.12 .29 \\
364 \\
\end{array}$ & $1118-1123$ & $\begin{array}{c}\mathrm{F}, \mathrm{H} \\
\text { IIIGG, II }\end{array}$ & - & $\begin{array}{l}-0.38 \pm 0.03 \\
-0.40 \mid-0.35\end{array}$ & $154-106$ & $\begin{array}{c}1.83 \pm 0.11 \\
-\mid 1.83\end{array}$ \\
\hline 56 & $\begin{array}{c}92.12 .30 \\
365 \\
\end{array}$ & $1034-1039$ & $\begin{array}{c}\mathrm{F} \\
\text { IIIG, IV, II }\end{array}$ & $0.32 \pm 0.08$ & $-0.071 \pm 0.004$ & $64-49$ & \\
\hline 57 & $\begin{array}{c}93.01 .01 \\
1 \\
\end{array}$ & $1344-1348$ & $\begin{array}{c}\mathrm{F} \\
\mathrm{III}, \mathrm{IV}, \mathrm{II}, \mathrm{RS}\end{array}$ & $0.42 \pm 0.17$ & $-0.25 \pm 0.01$ & $128-63$ & - \\
\hline 58 & $\begin{array}{c}93.01 .15 \\
15 \\
\end{array}$ & $0927-0930$ & $\begin{array}{c}\text { F? } \\
\text { IV, II }\end{array}$ & $0.34 \pm 0.06$ & $-0.37 \pm 0.06$ & $165-115$ & - \\
\hline 59 & $\begin{array}{c}93.04 .30 \\
120 \\
\end{array}$ & $1428-1432$ & $\begin{array}{l}\text { F } \\
\text { II }\end{array}$ & $0.24 \pm 0.04$ & $-0.068 \pm 0.004$ & $54-45$ & - \\
\hline 60 & $\begin{array}{c}93.05 .25 \\
145 \\
\end{array}$ & $1514-1517$ & $\begin{array}{l}\mathrm{F} \\
\mathrm{II} \\
\end{array}$ & $0.27 \pm 0.02$ & $-0.060 \pm 0.005$ & $53-48$ & $一$ \\
\hline 61 & $\begin{array}{c}93.06 .07 \\
158 \\
\end{array}$ & $1425-1435$ & $\mathrm{~F}, \mathrm{H}$ & $\begin{array}{c}0.49 \pm 0.16 \\
0.42 \mid 0.51\end{array}$ & $\begin{array}{l}-0.06 \pm 0.03 \\
-0.07 \mid-0.05\end{array}$ & $50-43$ & $\begin{array}{r}2.12 \pm 0.07 \\
\cdots \mid 2.12\end{array}$ \\
\hline 62 & $\begin{array}{c}93.07 .22 \\
203 \\
\end{array}$ & $0853-0903$ & $\mathrm{~F}, \mathrm{H}$ & $\begin{array}{c}0.31 \pm 0.10 \\
-\mid 0.31\end{array}$ & $\begin{array}{l}-0.05 \pm 0.04 \\
-0.05 \mid-0.05\end{array}$ & $66-48$ & $\begin{array}{c}2.11 \pm 0.10 \\
-\mid 2.11 \\
\end{array}$ \\
\hline 63 & $\begin{array}{c}93.09 .27 \\
270 \\
\end{array}$ & $1210-1218$ & $\begin{array}{c}\mathrm{F}, \mathrm{H} \\
\mathrm{IIIG}, \mathrm{II}, \mathrm{DC}\end{array}$ & $\begin{array}{c}0.38 \pm 0.02 \\
0.36 \\
0.40\end{array}$ & $\begin{array}{l}-0.23 \pm 0.02 \\
-0.22 !-0.25 \\
\end{array}$ & $136-54$ & $\begin{array}{l}1.95 \pm 0.11 \\
2.02 \mid 1.87\end{array}$ \\
\hline 64 & $\begin{array}{c}93.12 .27 \\
361 \\
\end{array}$ & $0915-0918$ & $\begin{array}{c}\mathrm{F}, \mathrm{H} \\
\mathrm{IIIG}, \mathrm{II}\end{array}$ & $\begin{array}{c}0.20 \pm 0.06 \\
0.16 \mid 0.24\end{array}$ & $\begin{array}{l}-0.20 \pm 0.05 \\
-0.16 \mid-0.25\end{array}$ & $134 \cdot 114$ & $\begin{array}{l}1.98 \pm 0.08 \\
2.03 \mid 1.92\end{array}$ \\
\hline 65 & $\begin{array}{c}93.12 .28 \\
362\end{array}$ & $1212-1216$ & $\begin{array}{c}\mathrm{F}, \mathrm{H} \\
\mathrm{IIIG}, \mathrm{II}\end{array}$ & $\begin{array}{c}0.67 \pm 0.12 \\
-\mid 0.67 \\
\end{array}$ & $\begin{array}{l}-0.38 \pm 0.08 \\
-0.46 \mid-0.30\end{array}$ & $120-65$ & $\begin{array}{l}1.99 \pm 0.11 \\
2.07 \mid 1.91 \\
\end{array}$ \\
\hline & \multicolumn{2}{|c|}{ average } & & $\begin{array}{c}0.32 \pm 0.14 \\
0.26 \mid 0.41\end{array}$ & $\begin{array}{l}-0.16 \pm 0.11 \\
-0.16 \mid-0.17\end{array}$ & & $\begin{array}{c}2.01 \pm 0.11 \\
2.04 \mid 1.95\end{array}$ \\
\hline & & & & fund. harm. & fund. / harm. & & upper / lower \\
\hline
\end{tabular}




\section{Statistical analysis}

The instantaneous bandwidths (cf. Eq. (1)) of the fundamental and harmonic bands of the type II bursts lie in the interval $0.07 \leq\left\langle(\Delta f / f)_{\mathrm{F}}\right\rangle \leq 0.59$ with a mean value of $\left\langle\left\langle(\Delta f / f)_{\mathrm{F}}\right\rangle\right\rangle=0.26$ and $0.07 \leq\left\langle(\Delta f / f)_{\mathrm{H}}\right\rangle \leq 0.74$ with a mean value of $\left\langle\left\langle(\Delta f / f)_{\mathrm{H}}\right\rangle\right\rangle=0.41$, respectively. The corresponding histograms are presented in Figs. 3 and 4. Combining the instantaneous bandwidths of both the fundamental and harmonic bands, a mean value and a standard deviation

$$
\langle\langle(\Delta f / f)\rangle\rangle=0.32 \pm 0.14
$$

is obtained with the distribution given in the histogram of Fig. 5.

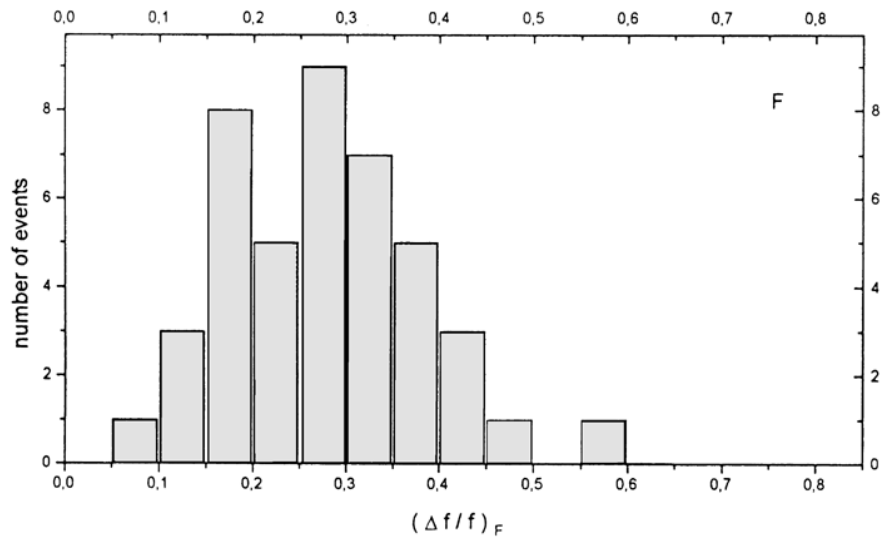

Fig. 3. Histogram of the mean instantaneous bandwidth $\left\langle(\Delta f / f)_{\mathrm{F}}\right\rangle$ of the fundamental band of all solar type II radio bursts analyzed (cf. Table 2)

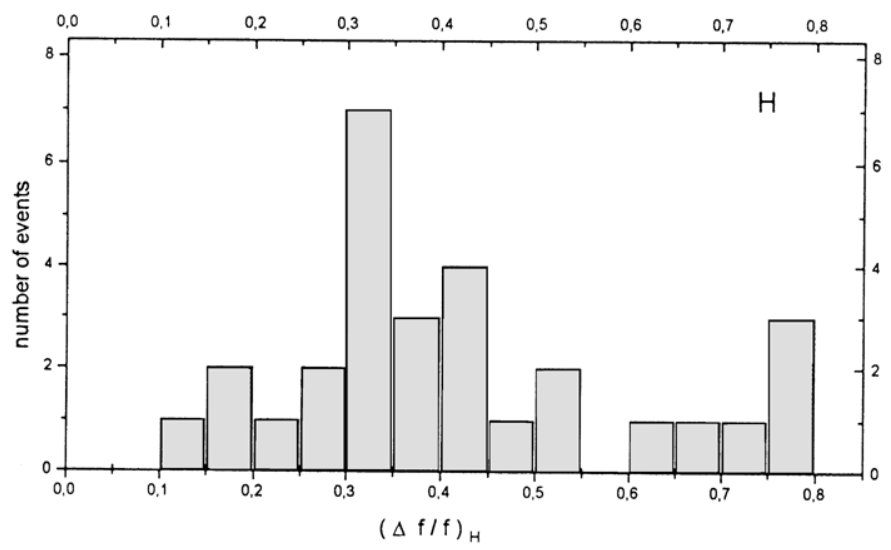

Fig. 4. Histogram of the mean instantaneous bandwidth $\left\langle(\Delta f / f)_{\mathrm{H}}\right\rangle$ of the harmonic band of all solar type II radio bursts analyzed (cf. Table 2)

Generally, the mean value and standard deviation of the drift rate averaged over the whole set of solar type II

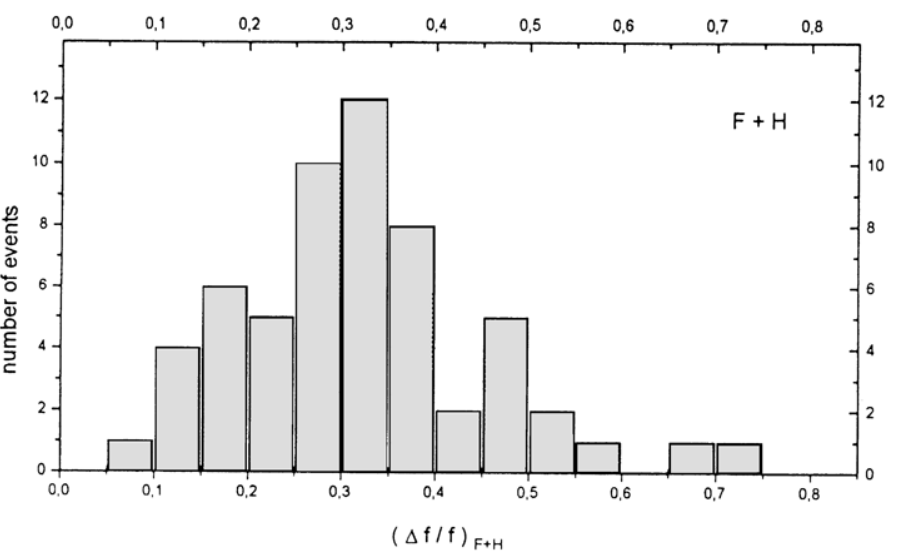

Fig. 5. Histogram of the instantaneous bandwidth of the fundamental and harmonic bands of all solar type II radio bursts analyzed (cf. Table 2)

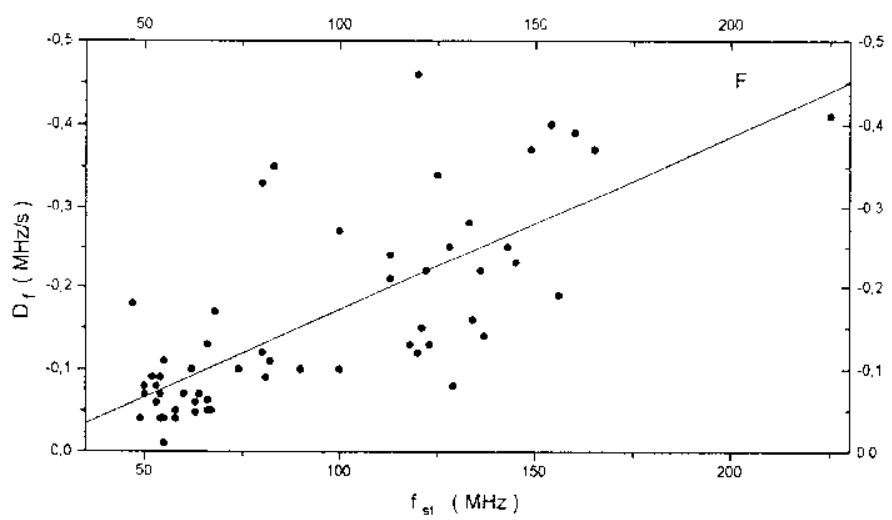

Fig. 6. Scatter plot of the magnitude of drift rate $-D_{f, \mathrm{~F}}$ versus the starting frequency $f_{\text {st }}$ for the fundamental band of all individual solar type II radio bursts analyzed. The straight line results from a linear regression analysis with a correlation coefficient of 0.738

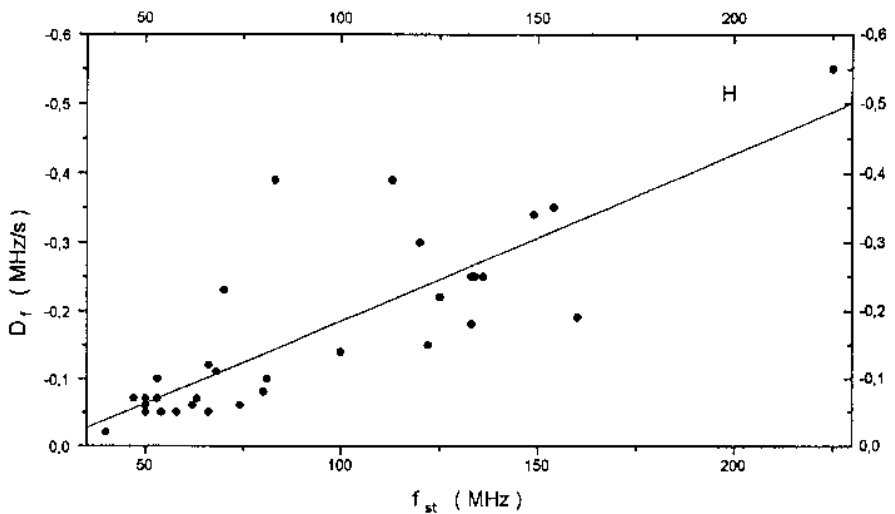

Fig. 7. Scatter plot of the magnitude of drift rate $-D_{f, \mathrm{H}}$ versus the starting frequency $f_{\text {st }}$ of the harmonic band of all individual solar type II radio bursts analyzed. The straight line results from a linear regression analysis with a correlation coefficient of 0.882 


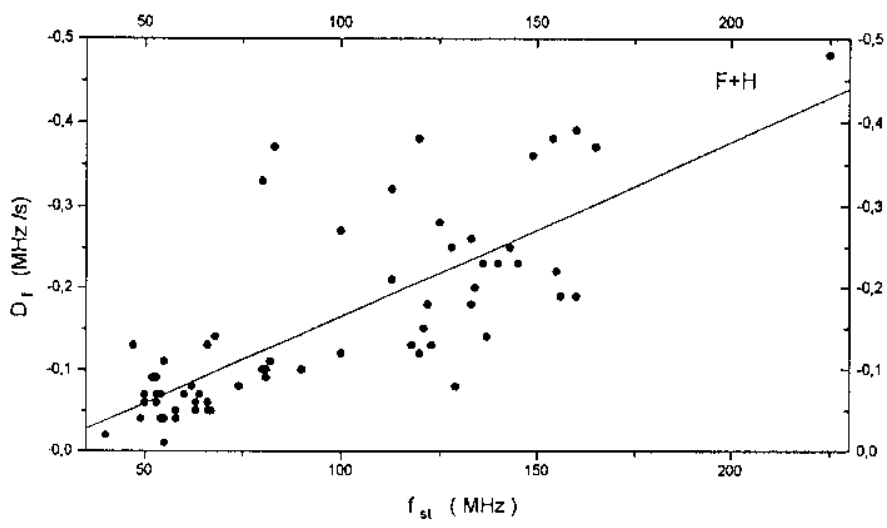

Fig. 8. Scatter plot of the magnitude of drift rate $-D_{f, \mathrm{~F}+\mathrm{H}}$ versus the starting frequency $f_{\text {st }}$ of both the fundamental and harmonic bands of all individual solar type II radio bursts analyzed. The straight line results from a linear regression analysis with a correlation coefficient of 0.779

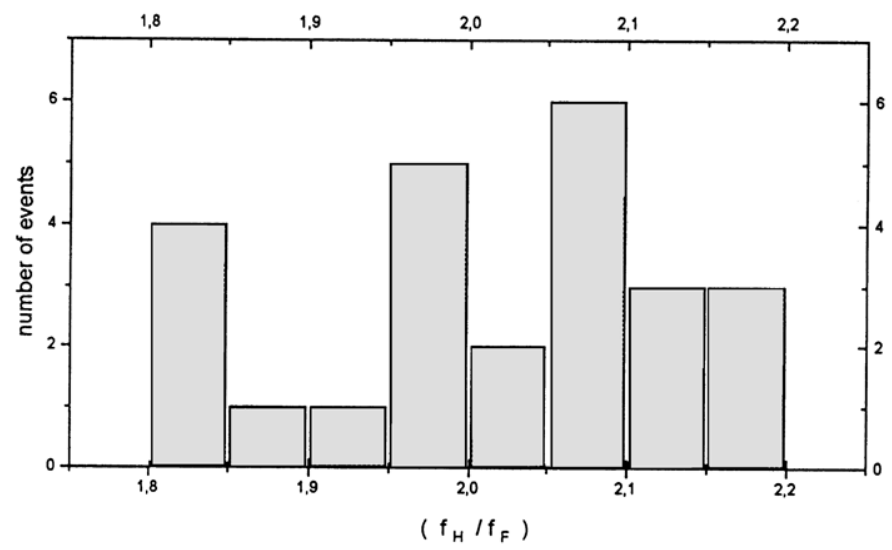

Fig. 9. Histogram of the mean values of the ratio $\left\langle f_{\mathrm{H}} / f_{\mathrm{F}}\right\rangle$ (cf. Eq. (3)) between the middle frequency of the harmonic and the fundamental band of the solar type II radio bursts analyzed (cf. Table 2)

radio bursts is found to be

$$
\left\langle D_{f}\right\rangle=-0.16 \pm 0.11 \mathrm{MHz} / \mathrm{s}
$$

It has been shown that the magnitude of the drift rate increases with the starting frequency of type II bursts (cf. e.g. Urbarz 1990; Mann 1995). This property of type II bursts is confirmed by this study. The corresponding scatter plots of the magnitude of the drift rate $-D_{f}$ versus the starting frequency $f_{\text {st }}$ of the individual bursts are presented for the fundamental and harmonic bands as well as for both data sets in the Figs. 6-8, respectively. Note that the half of the originally observed starting frequency has been used for $f_{\mathrm{st}}$ in the case of the harmonic bands. The linear regression analysis of the three data sets, i.e., $\left\{-D_{f, \mathrm{~F}}, f_{\mathrm{st}}\right\},\left\{-D_{f, \mathrm{H}}, f_{\mathrm{st}}\right\},\left\{-D_{f, \mathrm{~F}+\mathrm{H}}, f_{\mathrm{st}}\right\}$ provides

$$
\left(-D_{f, l}\right)=A_{l}+B_{l} f_{\mathrm{st}}
$$

$(l=\mathrm{F}, \mathrm{H}, \mathrm{F}+\mathrm{H})$ with $A_{\mathrm{F}}=-0.043 \mathrm{MHz} / \mathrm{s}, A_{\mathrm{H}}=-0.058$ $\mathrm{MHz} / \mathrm{s}, A_{\mathrm{F}+\mathrm{H}}=-0.046 \mathrm{MHz} / \mathrm{s}, B_{\mathrm{F}}=0.002 \mathrm{~s}^{-1}, B_{\mathrm{H}}=$ $0.002 \mathrm{~s}^{-1}$, and $B_{\mathrm{F}+\mathrm{H}}=0.002 \mathrm{~s}^{-1}$ (cf. the straight lines in Figs. $6-8)$. Here, the data set $\left\{-D_{f, \mathrm{~F}+\mathrm{H}}, f_{\mathrm{st}}\right.$ includes all drift rates of the fundamental and harmonic bands.

The analysis of the ratio $\left\langle f_{\mathrm{H}} / f_{\mathrm{F}}\right\rangle$ as defined by Eq. (3) provides the distribution presented in Fig. 9. The values of $\left\langle f_{\mathrm{H}} / f_{\mathrm{F}}\right\rangle$ lies in the interval $1.80 \leq\left\langle f_{\mathrm{H}} / f_{\mathrm{H}}\right\rangle \leq 2.20$ with a mean value and a standard deviation of

$$
\left\langle\left\langle f_{\mathrm{H}} / f_{\mathrm{F}}\right\rangle\right\rangle=2.01 \pm 0.11
$$

As the Fig. 9 shows, the distribution is sharply localized around this mean value. This result slightly differs from those in previous papers (cf. e.g. Nelson \& Melrose 1985). This difference is caused by the different methods of computing this ratio. In this paper the center- frequency of each emission band is used for calculating this ratio in contrast to the low- frequency edge of these bands as done in previous papers (cf. Maxwell \& Thompson 1962). Taking into account this difference the result of this paper agrees well with that of Maxwell \& Thompson (1962).

\section{Final remarks}

In this paper a catalogue of solar type II radio bursts observed during the time period September 1, 1990 until December 31, 1993 (cf. Table 1) is presented with several spectral properties (cf. Table 2) of each individual burst. A statistical analysis of these spectral properties has been performed (cf. Sect. 3). An interpretation of the statistical study of the instantaneous bandwidth has been given by Mann et al. (1995). They concluded that solar type II radio bursts are generated by supercritical, quasi-parallel, fast magnetosonic shocks travelling through the corona.

The observations were done during the first part of the ULYSSES mission. As mentioned above solar type II radio bursts are associated with CME's and interplanetary shocks (cf. Sect. 1), i.e., with large scale disturbances of the heliosphere. Such disturbances and associated particle events can be detected by the instruments aboard the ULYSSES satellite. Thus, the results presented in this paper will be useful for the investigation of solar related phenomena in the heliosphere by means of the data recorded by the ULYSSES satellite.

Acknowledgements. The authors are indebted to M.D. Dryer, K.-L. Klein, and P. Zlobec for stimulating discussions.

\section{References}

Aurass H., 1992, Ann. Geophys. 10, 359

Aurass H., Klein K.-L., Mann G., 1995, ESA-Journal, (in press)

Benz A.O., Thejappa G., 1988, A\&A 202, 267

Bougeret J.L., King J.H., Schwenn R., 1984, Solar Phys. 90, 401 
Bougeret J.L., 1985, in: Tsurutani B.T., Stone R.G. (eds.) Collisionless Shocks in the Heliosphere: Reviews of Current Research, AGU GN-34, Washington DC, p. 13

Cairns J.H., Robinson R.D., 1987, Solar Phys. 111, 365

Cane H.V., Stone R.G., Fainberg J., Steinberg J.L., Hoang S., 1982, Solar Phys. 78, 187

Dryer M., 1982, Space Sci. Rev. 33, 233

Ginzburg V.L., Zheleznyakov V.V., 1958, Sov. Astron. AJ 3, 235

Gopalswamy N., Kundu M.R., 1992, in: Zank G.P., Gaisser T.K. (eds.) Particle Acceleration in Cosmic Plasmas, American Institute of Physics, New York, p. 257

Gosling J.T., Hildner F., MacQueen R.M., Munroe R.H., Poland A.I., Ross C.L., 1976, Solar Phys. 48, 389

Holman G.D., Pesses M.E., 1983, ApJ 267, 837

Kliem B., Krüger A., Treumann R.A., 1992, Solar Phys. 140, 149

Krüger A., 1979, Introduction to Solar Radioastronomy and Radio Physics. Reidel, Dordrecht

Lengyel-Frey D., Stone R.G., 1989, J. Geophys. Res. 94, 159

MacQueen R.M., 1980, Phil. Trans. R. Soc. London, Ser. A 297, 605

Mann G., 1995, in: Benz A.O., Krüger A., Coronal Magnetic energy Release, Lecture Notes in Physics. Springer, Heidelberg, (in press)

Mann G., Aurass H., Voigt W., Paschke J., 1992, ESA-Journal, SP-348, 129

Mann G., Lühr H., 1994, ApJS 90, 577
Mann G., Classen T., Aurass H., 1995, A\&A (in press)

Maxwell A., Dryer M., McIntosh P., 1985, Solar Phys. 97, 401

Maxwell A., Thompson A.R., 1962, ApJ 135, 138

McLean D.J., 1967, Austr. J. Phys. 1, 47

Nelson G. S., Melrose D.R., 1985, in: McLean D.J., Labrum N.R. (eds.) Solar Radiophys. Cambridge Univ. Press, Cambridge, UK, p. 333

Nelson G.J., Robinson R.D., 1975. Proc. Astron. Soc. Aust. 2, 3670

Roberts J.A., 1959, Austr. J. Phys. 12, 327

Robinson R.D., Stewart R.T., Cane H.V., 1984, Solar Phys. 91, 159

Sheeley N.R., Stewart R.T., Robinson R.D., Howard R.A., Koomen M.J., Michels D.J., 1984, ApJ 279, 839

Smerd S.F., Sheridan K.V., Stewart R.T., 1975, ApJ Lett. 16, 23

Stone R.G., Cane H.V., Bougeret J.-L., 1984, in: Shea M.A. et al. (eds.) STIP Symposium on Solar Interplanetary Intervals, Engineering International, Huntsville, p. 371

Stone R.G., et al., 1992, A\&AS 92, 291

Urbarz H., 1990, report UAG-98 (National Geophysical Data Center), Boulder 1990

Wagner W.J., 1980, Adv. Space Res. 2, 203

Wild J.P., McCready L.L., 1950, Austr. J. Sci. Res. Ser. A3, 387

Wild J.P., Smerd S.F., 1972, ARA\&A 10, 159

Zheleznyakov V.V., 1965, Sov. Astron. AJ. 9, 191

Zaitsev V.V., 1966, Sov. Astron. AJ. 9, 572 